\title{
DOSSIER TENS-SCL 2012 \\ EL LILD Y LAS ESTRUCTURAS AUTOPORTANTES DE BAMBÚ PARA ARQUITECTURA TEXTIL
}

JOÃo CORREIA DE MELO

JOSÉ LUIZ RIPPER

LUÍS MOREIRA

LUCAS RIPPER

PROFESOR, LABORATÓRIO DE INVESTIGAÇÃO EM LIVRE DESENHO, DEPARTAMENTO DE ARTES E DESIGN, PONTIFÍCIA UNIVERSIDADE CATÓLICA DO RÍO DE JANEIRO, RÍO DE JANEIRO, BRASIL.

PROFESOR, LABORATÓRIO DE INVESTIGACÃO EM LIVRE DESENHO, DEPARTAMENTO DE ARTES E DESIGN, PONTIFÍCIA UNIVERSIDADE CATÓLICA DO RÍO DE JANEIRO, RÍO DE JANEIRO, BRASIL.

PROFESOR, LABORATÓRIO DE SISTEMAS ESTRUTURAIS, ESCOLA DE ENGENHARIA, DEPARTAMENTO DE ENGENHARIA DE ESTRUTURAS, UNIVERSIDADE FEDERAL DE MINAS GERAIS, BELO HORIZONTE, BRASIL.

PROFESOR, LABORATÓRIO DE INVESTIGAÇÃO EM LIVRE DESENHO, DEPARTAMENTO DE ARTES E DESIGN, PONTIFÍCIA UNIVERSIDADE CATÓLICA DO RÍO DE JANEIRO, RÍO DE JANEIRO, BRASIL.

\section{INTRODUCCIÓN} miles y miles de años, se constituyó como un sistema de cubierta eficaz y sencillo, adaptable a diferentes ambientes físicos y geográficos (Hatton, 1979, Otto et al., 1979). Permite montajes y desmontajes constantes y el transporte de manera práctica y eficiente para responder tanto a situaciones de culturas nómades tradicionales, como a las de la vida moderna Las carpas tradicionales se generaban de manera similar a los procesos espontáneos naturales, es decir, consumiendo un mínimo posible de energía potencial existente en el espacio local. Hoy día nuestra sociedad técnico-científica utiliza este tipo de cubierta principalmente para

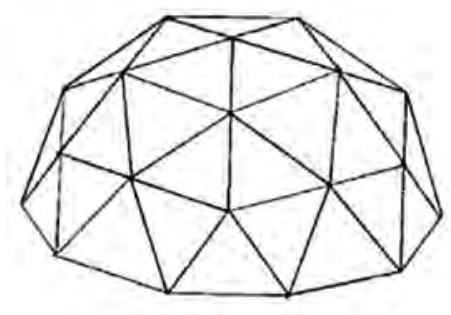

FIG 1 Solución de nudo industrial y solución de nudo artesanal desarrollado en el LILD, el "Giro".

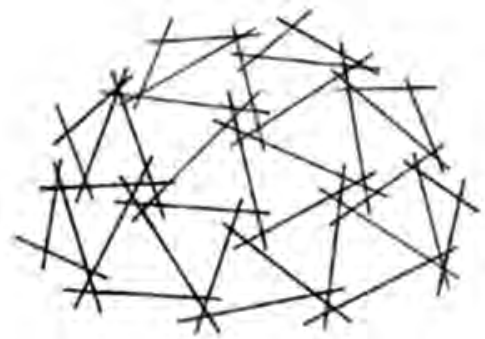

realizar megaestructuras. Sin embargo,
La carpa, como se la viene haciendo hace

se vienen aplicando en las más diversas culturas siguiendo objetivos y modalidades variadas. En Occidente se observa una tendencia a sumarlos a las técnicas industriales vigentes y a asociarlos con los materiales estandarizados presentes en la lógica de producción en serie. De esta manera, las potencias particulares de estos materiales de la naturaleza, que se presentan prácticamente listos para el uso, no se utilizan. Una vez estandarizados, estos materiales pasan a ser vistos y comprendidos de una misma manera y no ofrecen el desarrollo de sus potencialidades infinitas. Sin embargo, encontramos algunos nichos de aplicación e investigación que, del punto de vista ecológico y de la ciencia, tratan con buen comportamiento dichos materiales.

A continuación mostraremos algunos estas construcciones de gran tamaño y alto peso produjeron trajeron una sofisticación técnica innecesaria y de mayor costo a las carpas más pequeñas.

Con el objetivo de llegar a un punto medio entre estos dos momento históricos de las construcciones cubiertas con membrana, la investigación en el Laboratorio para Investigación en Libre Diseño de la Puc de Río busca unir el conocimiento lógico académico y la sabiduría popular espontánea de manera de difundir y popularizar estructuras liviana idóneas con posibilidad de construirse artesanalmente en ambientes comunitarios.

En sus alrededor de treinta años el LILD, antiguo LOTDP, y su nuevo colaborador LASE - Laboratorio de Sistemas Estructurales - EEUFMG, viene desarrollando técnicas adaptadas al bambú y a la tierra cruda en construcciones útiles, de bajo impacto y de carácter popular. Estos materiales naturales, de uso corriente en el mundo desde los tiempos remotos, capaces de interferir positivamente en el mismo cuando lo copian en el proceso de transmisión de la técnica (Campos y Melo, 2011; Correia de Melo, Yamaki, y Ripper, 2012).

Otro punto importante se refiere a la obtención de las formas que se utilizan en la investigación del LILD: siempre se busca observar el continuum informacional de lo que nos da la naturaleza (Correia de Melo, Ripper, y Yamaki, 2012). En conjunto con el investigador, o a falta del mismo, la naturaleza produce sus formas. Lo que el hombre intenta es cerrar en parámetros estas formas de manera de intentar reproducirlas en el ámbito de sus capacidades técnicas. No obstante, en gran parte de los casos, después de descubrir algunos de estos parámetros, el hombre los toma como verdades irrefutables. Eso paraliza la técnica, lo que limita las posibilidades del trabajo conjunto con la naturaleza.

Por lo tanto, en el laboratorio se busca que el investigador sea destituido de los conceptos técnicos previos a través de la experimentación práctica en objetos puestos en nuestra realidad concreta, ya sean modelos reducidos, modelos en escala real $\mathrm{u}$ objetos experimentales en estado de uso (Ripper y Moreira, 2004; Campos y Melo, 2011; Correia de Melo, Ripper, y Yamaki, 2012; Ripper, Campos, y Correia de Melo, 2012, Yamaki, 2012). Esta práctica es más impulsada cuando la experimentación se hace con elementos que presentan una forma natural, como en el caso de burbujas de jabón y modelos funiculares (Otto y Rasch, 1995).

Cabe recordar que esta metodología es resultado del proceso de trabajo sujeto al desarrollo técnico y al constante aprendizaje con las formas naturales y, como tal, está en continua transformación. La metodología se viene demostrando bastante efectiva y, además del LILD, se está aplicando con éxito en el LASE (Moreira, Ripper, y Caliman, 2010).

\section{AVANCES TÉCNICOS}

Las técnicas desarrolladas en el LILD no están dirigidas a la gran industria y tampoco al mercado de los objetos de uso corriente de la lógica fabril. El objetivo básico de la investigación sobre técnicas específicas para el bambú es desarrollar 


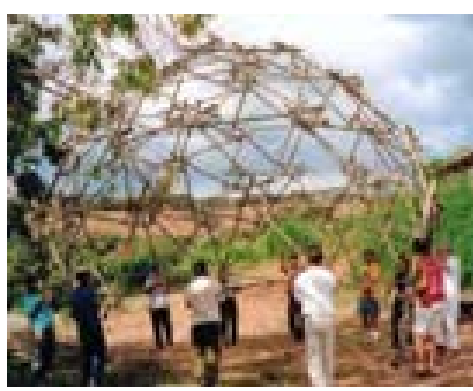

FIG 2 Estructura en celosía de bambú transportada por constructores locales.

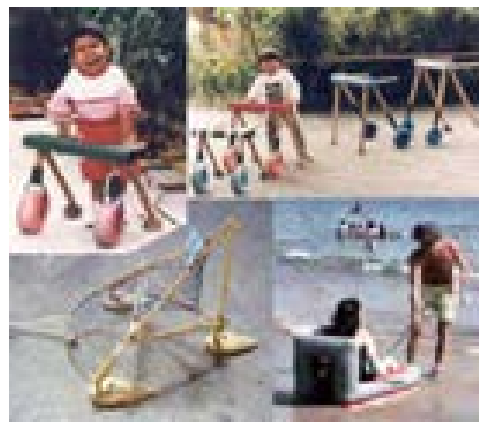

FIG 3 Andadores y vehículos anfibios.

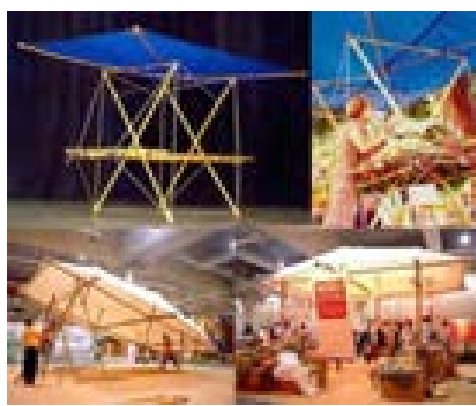

FIG 4 Autotensados.

Fuente: Mario Seixas, proyecto de graduación en Diseño, PUC-Rio, 2001; y BAMBUTEC, Río de Janeiro.

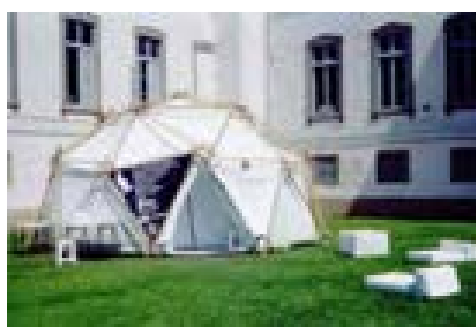

FIG 5 Domo Tensegrity.

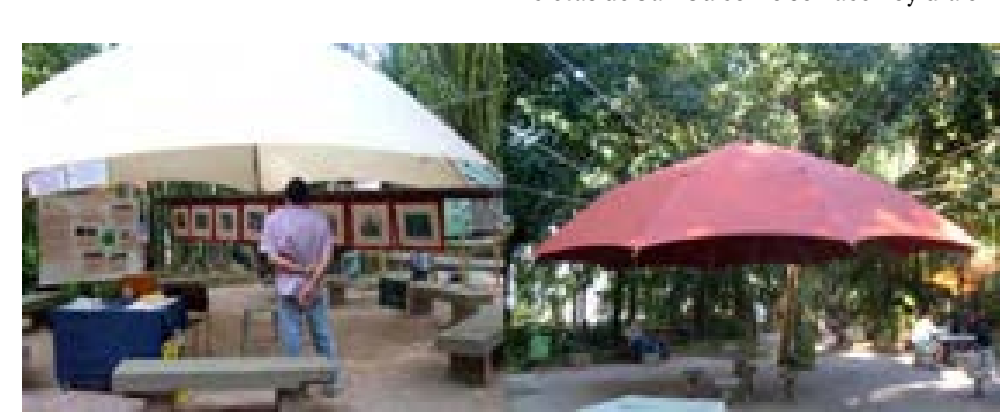

FIG 6 Sombrillas de bambú distribuidas por el campus de la PUC-Rio,

aprovechando la técnica milenaria china.

estructuras arquitectónicas que estén en consonancia y que establezcan una comunicación directa entre el LILD y las comunidades rurales y urbanas para la transferencia de tecnología y modos de construcción, desengañando los objetos y resignificando la dependencia existente entre estas comunidades y el mercado de la construcción civil.

Buckminster Fuller y Konrad Wachsmann, que trabajaron con estructuras con celosías hechas en perfiles metálicos o de madera industrializados, solucionaron de diferentes maneras el problema de los nudos que conectaban las extremidades de las diversas barras convergentes a un punto central. Sin embargo, estas soluciones industriales son inadecuadas para el bambú cuando el objetivo es simplificar y permitir la producción del objeto para la población. Debilidades debido a la microestructura fibrosa de esta planta tornan difícil la recepción de esfuerzos puntuales realizados por estos nudos.

En la investigación del laboratorio este problema se solucionó eliminando la necesidad de un elemento específico para el nudo. Para esto, se desarrollaron dos sistemas constructivos: el sistema tensegrítico y el sistema de atado (Ripper et al., 2014). Ambos solamente utilizaron elementos de bambús y cables sintéticos o naturales cubiertos por membrana, lo que hizo más fácil la técnica y su consiguiente apropiación por parte de las comunidades junto a la mano de obra local (fig. 1 y 2).

\section{AUTOPORTANTES Y AUTOTENSADOS}

Los trabajos sobre estructuras autoportantes de bambú tienen su origen en los equipos destinados a portadores con dificultades motoras (fig. 3). Estos equipos son vehículos de bambú livianos impulsados por la fuerza humana, hechos artesanalmente a medida, concretos y en continuo perfeccionamiento con técnicas especialmente adaptadas a las condiciones estructurales del bambú.

Los andadores de bambú ayudan a los niños con parálisis cerebral sin equilibrio, a caminar. Ellos se están construyendo de varios tipos y con distintas técnicas. Las articulaciones de los tipos mostrados se basaron en la articulación que Santos Dumont utilizaba para la pequeña cesta de sus balones y esta solución de articulación fue lo que posibilitó, más tarde, la producción de bicicletas de bambú como se hace hoy día en
Dinamarca. Estas articulaciones tienen cables tirados a lo largo del interior del tallo. Otro tipo de vehículo mostrado es el deslizador anfibio, que ayuda a trasladarse a personas que utilizan sillas de ruedas, donde estas no funcionan. La observación del funcionamiento de estos equipos confirma no solamente el potencial general de las técnicas utilizadas, sino que también mejoran la condición de autoportancia estructural resultante de los desplazamientos, a veces violentos, de los vehículos.

El conocimiento adquirido con estos experimentos abre camino para nuevas formulaciones de tipologías estructurales favorables a la autoportancia que, últimamente, se ha aplicado a objetos con mayores dimensiones. Estas son estructuras para edificaciones que se vienen realizando con las mismas condiciones de autoportancia estructural de los equipos mencionados, lo que apunta a cimientos superficiales, es decir, solamente posados en la superficie del terreno.

Estas estructuras -conocidas como núcleos de resistencia-no necesitan de los cimientos usuales o de bloques pesados a cielo abierto para anclaje y se vienen utilizando para tensionar membradas de cubierta y para apoyar cáscaras estructurales, entre otros medios de sellado y de climatización interna.

Mayoritariamente, las formas de estas estructuras no son aquellas producidas por la imaginación del hombre. Para describir sus geometrías se utilizan formas derivadas de aquellas descubiertas en la naturaleza y obtenidas por dispositivos puestos en interacción con determinados fenómenos naturales como, por ejemplo, la gravedad, las presiones hidrostáticas, las tensiones superficiales de los líquidos, entre otros (Ripper y Moreira, 2004; Moreira, 2008; Correia de Melo, Ripper y Moreira, 2013) También se utilizan formas derivadas de polígonos pitagóricos de caras iguales y estables, como el icosaedro.

\section{OBJETOS DESARROLLADOS EN MEDIO} CULTURAL

En la actualidad, se está posibilitando el desarrollo de diversos tipos de estructuras experimentales auto portantes de bambú que puedan servir a edificaciones multiuso tales como salones de clase, salas de reunión, salas de lectura, bibliotecas, espacios de exposición para eventos, talleres y restaurantes entre otros. Para una mejor ejemplificación, a continuación se presentan algunos de estos objetos originados de la investigación de laboratorio y su uso en el medio cultural.

\section{AUTOTENSADOS}

Usualmente, los objetos más pequeños nos enseñan de mejor manera en comparación con aquellos más grandes. Esto se debe a las relaciones entre el hombre y el objeto: en relación a los más pequeños somos gigantes, los dominamos; mientras que en relación a los más grandes, nos sentimos dominados. La carpa tensada -proyecto de graduación del diseñador Mário Seixas, PUC de Río, 2011 (fig.
4)- se hizo para una feria de productos naturales, lo que demuestra una vez más la versatilidad, eficiencia, levedad y sencillez mecánica de este tipo de estructura prácticamente desconocida por las instituciones de enseñanza e investigación y también por los profesionales que lidian con los objetos de obra humana.

Por ocasión de la feria de productos orgánicos en São Paulo, la empresa BAMBUTEC (colaboradora del LILD) desarrolló una estructura tensada hecha con culmos de bambús y cables sintéticos que sirvieron de stand de presentación comercial. La conjugación de los elementos rígidos con la red de cables fue especialmente estudiada para permitir los tiempos de montaje disponibles en el evento, considerando cuatro personas especializadas a cargo de su levantamiento. En ese sentido, los planes de montaje deben ser continuamente mejorados y estas estructuras auto portantes se muestran bastante adecuadas para estos fines. Asimismo se hizo un análisis estructural del objeto que, de esta manera, representa la intensa colaboración en el desarrollo de objetos entre el LILD, el LASE y la BAMBUTEC (Moreira, Ripper y Caliman, 2010).

\section{STAND DE EXPOSICIÓN - DOMO TENSADO}

Alrededor del año 2000 un trabajo de magíster en Artes y Diseño de la Puc de Río realizó una estructura cupular hecha de bambú del tipo philostachys aurea e inscrita en un hemisferio aproximado de 8 metros de diámetro por 3,8 metros de altura. El objetivo fue crear una sala totalmente oscura que se debía montar en diversas localidades de la ciudad de Río de Janeiro para realizar determinados experimentos públicos (fig. 5).

Gracias a esta oportunidad se pueden conocer las posibilidades prácticas de este tipo de estructura: rapidez y facilidad de montaje, desmontaje y almacenamiento; posibilidad de transporte en vehículos sencillos con rack. En este tipo de estructura tensada cada bambú tiene su cable adjunto de manera semejante a un arco y flecha. La membrana de cubierta es colgada y tirada en la estructura cupular (Leite, 2003).

\section{SOMBRILLA}

En 2003 se buscaba una cubierta para las estructuras de los domos geodésicos de bambú que se perfeccionaban en el laboratorio, porque, en ese momento, se pasaba a considerar estos objetos como núcleos de resistencia y no solamente como una forma emblemática considerada socialmente. Uno de los objetos observados para tal objetivo fueron las milenarias sombrillas chinas hechas de bambú y papel de arroz que, vinculadas al domo, cubrirían el espacio definido. Las sombrillas llamaron la atención por su delicadeza, simplicidad estructural y funcionalidad. La facilidad de acoplamiento en el domo se demostró al fijarse en el modelo hecho a medida. Después de algunos estudios en modelos reducidos y luego de la determinación del modelado del tejido empleado, se hicieron 
FIG 7 Carpa de bambú y membrana textil, cubierta del nuevo LILD.

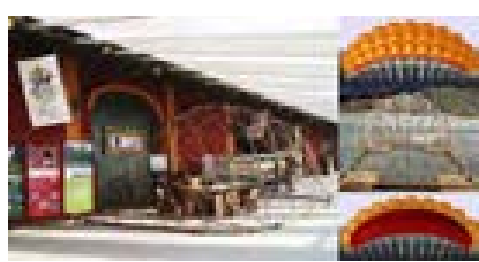

FIG 8 Bubble hall en la Río+20 y mejorías previstas.
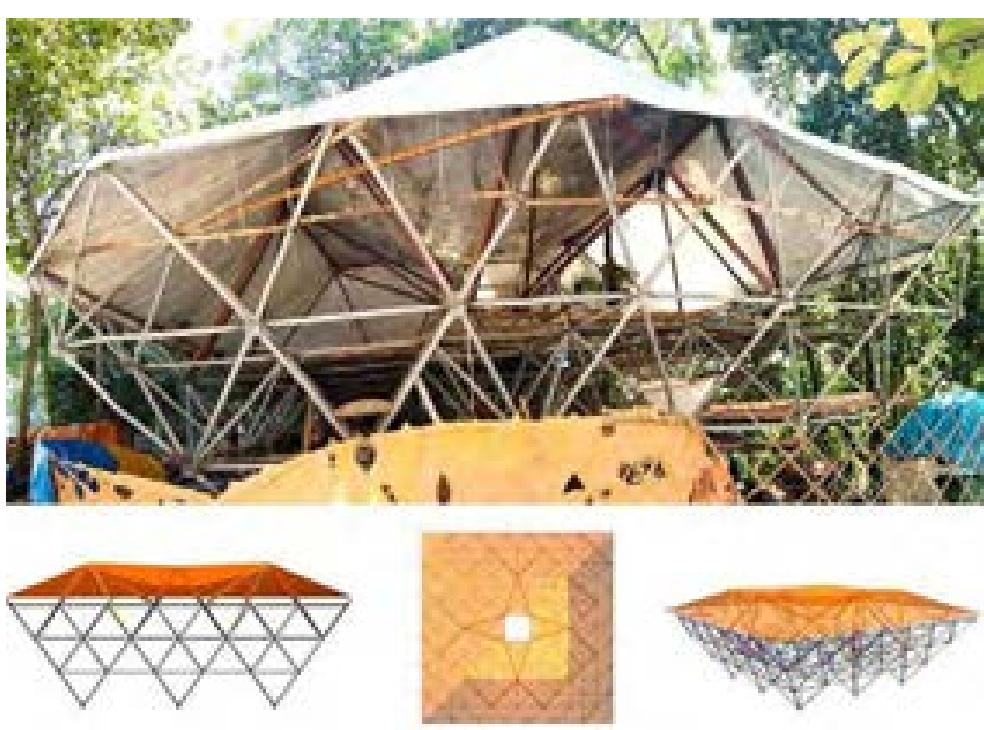

tres objetos experimentales con cinco y seis gajos. Estos modelos, en estado de uso, utilizaban tallos de bambú como astas, junta central de acero y membrana de tejido de algodón (fig. 6).

Las sombrillas se instalaron en el jardín del campus de la PUC de Río, fueron instaladas en el aire por medio de cables tensores fijados en los árboles, probando de esa manera, sus condiciones de usabilidad y comportamiento aerodinámico. Permaneciendo en el sitio por algunos meses, la sombrilla resistió vientos fuertes $y$, debido a sus condiciones de anclaje, se movía con el viento en la vertical sin afectar su estructura (Ripper y Moreira, 2006; Moreira y Ripper, 2006)

CARPA DE BAMBÚ Y MEMBRANA TEXTIL - UNA CAMINADA POR EL MÉTODO DE DISEÑO

A mediados de 2007, con motivo del cambio de ubicación del laboratorio, se desmontó la estructura de elementos tubulares de acero en celosía que tenía el LILD y se volvió a montar en otro lugar de la PUC de Río. Faltó proyectar la cubierta de los niveles superiores y sellados para dar cuenta de la nueva configuración que se hizo por el remontaje de la celosía que quedó como una pirámide truncada invertida con $10 \mathrm{~m}$ de base y $17,5 \mathrm{~m}$ en su plan superior. En el caso de la cubierta, una carpa de bambú y lona plástica se debería instalar en el plan superior de la celosía (fig. 7).

En las investigaciones sobre la forma de la cubierta se utilizaron experimentos con redes colgadas, funiculares y con burbujas de jabón, con un enfoque principal en estas últimas. Estas se soplaron en superficies planas y fueron manipuladas por los investigadores para cumplir con determinados parámetros establecidos tales como perímetros, alturas y otros. Luego se establecieron los límites de la forma, perímetro del plan superior y agujero central para ventilación e iluminación. La burbuja de jabón, conducida por estos parámetros, determinó un semi-toro de base cuadrada con curvaturas de la superficie determinada por la superficie mínima de la misma. Basándose en esta forma se hizo un gran modelo con estructura triangulada de bambú.
Durante la observación de esto modelo parecía que algo faltaba o sobraba. Había muchos bambús en la estructura lo que la hizo densa ya que contaba con 96 piezas de seis tamaños diferentes. En aquel momento los bambús destinados a la cubierta estaban alineados de pie a 6 $m$ de altura en la gran pared de la cancha deportiva que se ocupaba provisoriamente. En este punto una surgió pregunta: ¿por qué cortarlos? ¿por qué no utilizarlos de esta manera? Se descartó, entonces, el modelo pacientemente producido y, modificándose la geometría general, los grandes bambús de $6 \mathrm{~m}$ se utilizaron enteros, ordenados según las líneas generales de la forma que la burbuja presentaba, lo que redujo los elementos de la estructura a 36 piezas iguales (Ripper, Campos y Correia de Melo, 2012).

\section{OBJETOS EN MEDIO SITUACIONAL}

En este punto se presentan algunos objetos que están en fase de experimentación en el medio situacional, es decir, en el laboratorio. Se están desarrollando los aspectos técnicos, matemáticos y mecánicos de acuerdo a necesidades preestablecidas que, posteriormente cuando sean accionadas por el medio cultural, volverán con informaciones más precisas sobre su real funcionamiento.

\section{BUBBLE HALL}

La sala burbuja surgió en una propuesta del laboratorio, aprobada posteriormente por la dirección de la PUC de Río, sobre el descubrimiento y experimentación de espacios no convencionales de enseñanza. De esta manera, se encuentra en fase de desarrollo la ejecución de una sala basada en la forma de una burbuja singular que emerge a partir de una base rectangular. La aproximación a la geometría de la burbuja fue obtenida por medio de diversos experimentos que utilizaron técnicas electrónicas y artesanales para lograr una cuadrícula de cintas de bambú que determinan la forma de dicha burbuja.

Una estructura piloto se montó en un evento de la Río+2O, conferencia de las Naciones Unidas sobre el desarrollo sustentable, donde se la utilizó para la exposición de trabajos del propio labo- ratorio y de la red mundial de bambú y ratán, el INBAR (fig. 8). El próximo paso es sellar la estructura de la sala hecha de finas láminas de fibrobarro ${ }^{1}$. Los experimentos del nuevo espacio se llevarán a cabo por medio de una serie de clases que serán programadas (Correia de Melo et al, 2014).

\section{ICOSAEDRO DE MÁSTILES}

Durante varios años la investigación se enfocó en el domo geodésico de frecuencia dos (fig. 9). Dicho domo es una derivación del icosaedro, un sólido platónico propuesto por Fuller. De esta manera una mayor cantidad de puntos es proyectada en la esfera, aproximando la geometría de la celosía a la de esta. Para cada domo 2V (como se conoce el icosaedro de frecuencia 2) se necesitan 55 barras de dos tamaños diferentes. Sin embargo, en el último tiempo, se viene descubriendo que las potencialidades del bambú, como material que la naturaleza nos ofrece prácticamente listo, se reducen a medida que se cortan los tallos: el ideal es utilizarlos en el mayor largo posible. Así, las atenciones se vuelven al icosaedro que, para montarse próximo a la semiesfera, necesita solamente 25 barras de igual tamaño.

En ese momento se pretende confeccionar un icosaedro con barras de bambú del mayor tamaño posible. No obstante, cuanto más grande la barra, más grande es su flexión, y una manera de solucionar esta desventaja es la trasformación de las mismas en mástiles auto tensados (Moreira, Castro y Ripper, 2004). Al observar estos mástiles se nota que la cruceta empleada para armarlos proyecta algunos puntos afuera de la estructura. De esa manera, se percibe que estos puntos de la cruceta pueden ser los puntos de la geodésica $2 \mathrm{~V}$ que se proyectan en la esfera. Entonces, se monta un icosaedro (1V) con una cubierta referente a un domo 2C (Ripper et al, 2013).

Tal como en la sala burbuja, esta geometría permite la construcción de una cobertura de doble capa, donde la de afuera es compuesta de un laminado impermeable y la de adentro de una fina cáscara de fibro-barro, lo que permite un mayor confort ambiental. El próximo paso es aplicar esta geometría al medio cultural.

\section{CUBIERTA DE FORMOSO}

La idea de producir esta estructura surgió de la necesidad de cubrir un ambiente construido en el campo experimental avanzado del laboratorio situado en el villorrio de Formoso, ubicado en el interior del estado de São Paulo, sureste brasileño. Una primera tentativa para la cubierta de este pequeño abrigo se hizo hace algunos años. En dicha ocasión se construyó una primera estructura tensada cubierta con una lona plástica. Esta estructura fue removida debido a la intemperie. El efecto del viento rompió la lona, no obstante, la estructura principal se mantuvo intacta, lo que resultó en una serie de observaciones para la ejecución de una nueva estructura. 


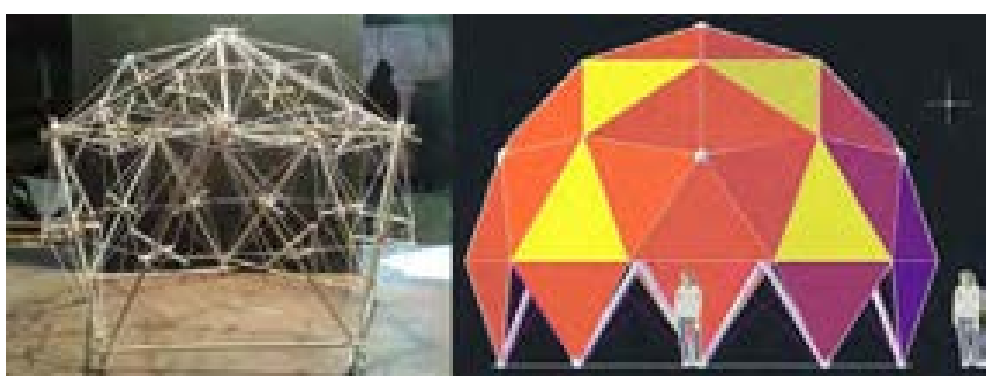

FIG 9 Icosaedro de mástiles, modelo en escala reducida y modelo 3D.

Material compuesto de tierra cruda $\mathbf{1}$ y fibras naturales desarrollado en el LILD (Leme, 2003).

El principio estructural del objeto es muy similar al de las estructuras tensadas. Sus elementos estructurales básicos son dos: elementos de compresión y elementos de tracción. La diferencia conceptual entre las estructuras tensadas y la que proponemos, está en la autonomía de sus juntas. Sin embargo, de manera general, la red continua de cables continúa trabajando a tracción y las vigas de bambú, básicamente, a compresión.

Las estructuras autoportantes y autotensadas están compuesta de tres elementos: vigas de bambú arqueadas, dores- $y$ red de cables. Sobre esta estructura básica se apoyan bandas de tejido natural y tierra. La longitud de estas bandas es variada y la sobreposición de las mismas permite suavizar la geometría pretendida. Después de montarla, la membrana se pinta con resina de origen vegetal. De esta manera se forma un compuesto maleable e impermeable.

Con vistas a mejorías futuras del objeto (fig. 10), se propone un reestudio tanto de la forma obtenida, como de la geomástiles de bambú -o elementos armametría de la red de cables que se utilizó. Para tal objetivo se pretende tomar como base las superficies mínimas formadas por las películas de jabón. Algunos estudios preliminares ya realizados, indicaron rumbos prometedores en dirección a la reducción del peso, materiales, mano de obra y tensiones en la membrana estructural (Correia de Melo, Stoffel y Ripper, 2014).

FIG 10 Modelo, montaje de la membrana aspecto final de la cubierta.

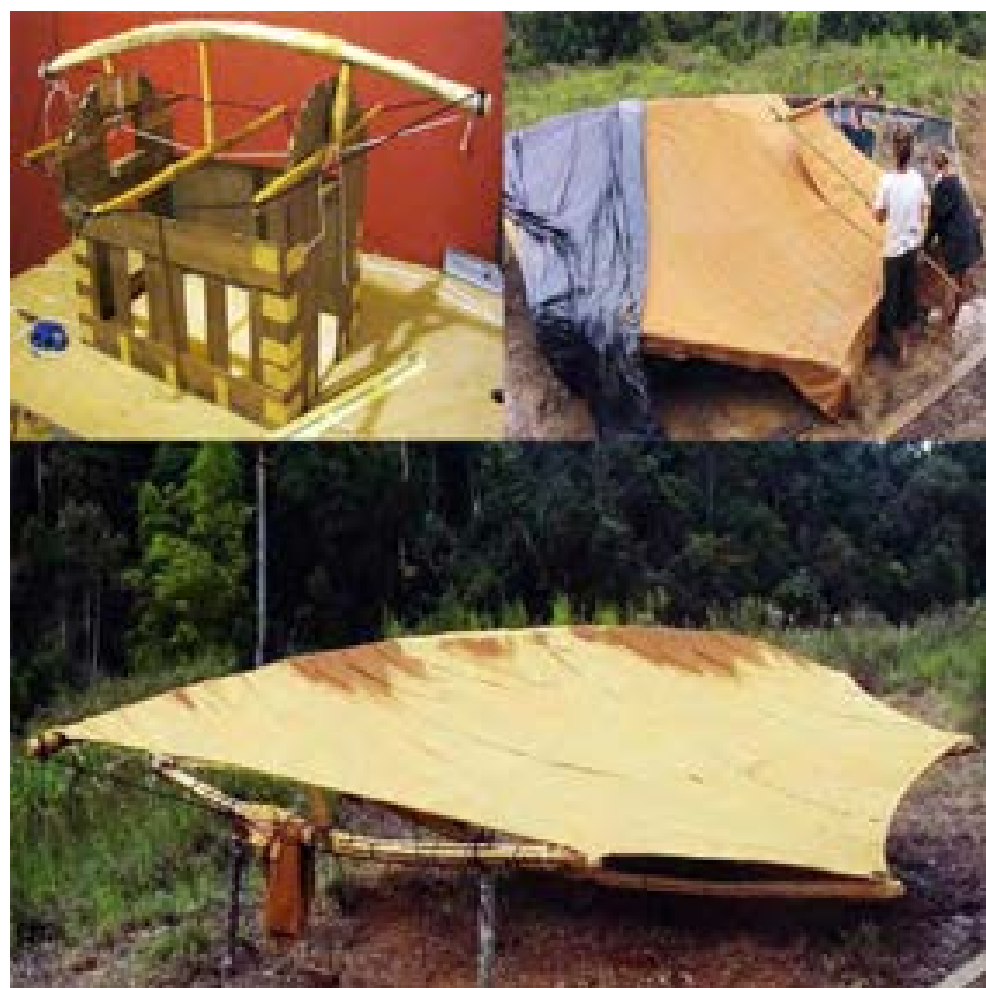

\section{CONSIDERACIONES FINALES}

A lo largo de la historia, las áreas de concepción y construcción de los objetos se vienen fragmentando y, cada vez más, pierden contacto entre sí. Así, se limitan a las visiones sobre tipologías estructurales y constructivas de determinadas áreas. El diseño trata sólo de pequeños objetos y la arquitectura lidia con grandes construcciones, del mismo modo que la ingeniería industrial concreta el primero y la ingeniería de estructuras, la segunda. Esta falsa linealidad crea nichos de actuación y dogmas constructivos que impiden las interacciones y nuevos abordajes sobre el objeto construido.

Por lo tanto, se propone una nueva visión sobre la concepción y construcción de los objetos tal como se puede ver en los ejemplos presentados. Diferente de los métodos vigentes, que transfieren la lógica de las grandes construcciones para los pequeños objetos, en el LILD se subvierte este orden: se enfoca en el pequeño para búsqueda de soluciones para el grande.

\section{AGRADECIMIENTOS}

Los autores agradecen a la BAMBUTEC, empresa de diseño y arquitectura colaboradora con sede en Río de Janeiro; y al LASE - EEUFMG por la colaboración en el desarrollo y análisis de objetos, además de la cesión de fotos para este artículo.

\section{BIBLIOGRAFÍA}

CAMPOS, D. M. y MELO, J. A. “Square-

based Bamboo Dome Based on Geodesics". En: Book of Abstracts. International Conference on NonConventional Construction Materials and Technologies IC-NOCMAT (décimo tercer versión, 2011, Changsha, China) CORREIA DE MELO, João. Modelos em linguagem mecânica e modelos em linguagem eletrônica: as interações na metodologia do LILD. Tesis de Magíster. Río de Janeiro, Pontificia Universidad Católica de Río de Janeiro, 2011. CORREIA DE MELO, João; RIPPER, José Luiz y MOREIRA, Luís. Development of bamboo structures based on minimal surfaces and natural formations. En: Symposium Beyond The Limits of Man, Proceedings of the International Association for Shell and Spatial Structures IASS (2013, Wroclaw, Polonia). CORREIA DE MELO, João; RIPPER, José Luiz; RIPPER, Lucas y TEIXEIRA, Walter. "The Bubble Hall: Bamboo reticular geodesic structure with the shape of soap bubble". Key Engineering Materials, 600: 78-86, febrero de 2014

CORREIA DE MELO, João; RIPPER, Jos Luiz y YAMAKI, Roberto Takao. Formfindin Process for Bamboo Structures. Ponencia presentada al Congreso Mundial de Bambú (2012, Antwerp, Bélgica), p. 12. CORREIA DE MELO, João; STOFFEL, Patrick y RIPPER, José Luiz. Módulo de Cobertura Têxtil com Estrutura Autoportante de Colmos de Bambu Amarrados. Ponencia presentada al Congreso Luso-Brasileiro de Materiais de Construção Sustentáveis (2014, Guimarães, Portugal), p. 10
CORREIA DE MELO, João; YAMAKI, Roberto Takao y RIPPER, José Luiz. Passagem de técnicas construtivas de bambu fibrobarro para comunidade de baixa renda. En: IV Congresso de Arquitetura e Construção com Terra no Brasil, (2012. Fortaleza, Brasil).

HATTON, E. The Tent Book. Boston, Houghton Mifflin Company, 1979 ILLICH, Iván. A convivencialidade. Lisboa, Publições Europa-América, 1976 LEITE, Claudia. Estética Sensorial Não Visual: Percepção do Belo Háptico. Tesis de Magíster. Río de Janeiro, Pontificia Universidad Católica de Río de Janeiro, 2003

LEME, Fernando. Construção com "fibrosolo": um estudo de caso sobre o resgate da técnica de taipa, e seus efeitos no ambiente de clima tropical úmido com estação seca e chuvas de verão. Tesis de doctorado. Río de Janeiro, Pontificia Universidad Católica de Río de Janeiro, 2003.

MOREIRA, Luís y RIPPER, José Luiz. Jogo das formas: a lógica do objeto natural. Río de Janeiro, Nau Editora, 2014. MOREIRA, Luís y RIPPER, José Luiz. Coberturas em Sombrinha. En: $X$ EBRAMEM-X Encontro Brasileiro em Madeiras e Estruturas de Madeira (2006, São Carlos, Brasil). MOREIRA, Luís; RIPPER, José Luiz y CALIMAN, Flávio. "Estruturas Autotensionadas com Bambus e Cabos Aplicadas a Coberturas Aéreas". Revista Gestão Universitária [en línea]. Marzo de 2010. Disponible en: < http:// estaouniversitaria com br/artioos/ estruturas-autotensionadas-com-bambuse-cabos>

OTTO, Frei. Arquitectura adaptable. Barcelona, Gustavo Gili, 1979. OTTO, Frei y RASCH, Bodo. Finding Form: Towards an Architecture of the Minimal. Michael Robinson (Trad.). Stuttgart, Axel Menges, 1995.

RIPPER, José Luiz y MOREIRA, Luís. Métodos de Ensino de Design de Produtos e sua Aplicação às Estruturas da Engenharia Civil. En: Congresso Brasileiro de Ensino de Engenharia, (2004, Brasilia, Brasil).

RIPPER, José Luiz y MOREIRA, Luís. "Sombrinhas de Bambu-Domínio Construtivo". En: International Conference on Non-Conventional Construction Materials and Technologies IC-NOCMAT (octava versión, 2006, Salvador, Brasil). RIPPER, José Luiz; CAMPOS, D., y CORREIA DE MELO, João. "TextileArchitecture Structured on Bamboo Culms". Key Engineering Materials, 517: 189-196, junio de 2012 RIPPER, José Luiz; MOREIRA, Luís; SILVA, M., CORREIA DE MELO, João y RIPPER, Lucas. "Domos Geodésicos de Bambu do Laboratório de Investigação em Livre Desenho - PUC-Río: Origens, Referências e Inovações em estruturas Autoportantes Não Convencionais". En: Anais do I CLB MCS. Guimarães, Universidade do Minho, 2014

YAMAKI, Roberto Takao. 0 uso da miniatura no desenvolvimento e passagem de formas técnicas: subjetividade e materialidade Tesis de Magíster. Río de Janeiro, Pontificia Universidad Católica de Río de Janeiro, 2012. 